OPEN ACCESS

Edited by:

Paola Marziani,

Osservatorio Astronomico di Padova

(INAF), Italy

Reviewed by:

Andjelka Branislav Kovacevic,

University of Belgrade, Serbia

Sven Zschocke,

Technische Universität Dresden,

Germany

${ }^{*}$ Correspondence:

Antonella Vallenar

antonella.vallenari@inaf.it

Specialty section:

This article was submitted to

Crop and Product Physiology,

a section of the journal

Frontiers in Astronomy and Space

Sciences

Received: 16 February 2018 Accepted: 03 April 2018

Published: 20 April 2018

Citation:

Vallenari A (2018) The Future of Astrometry in Space.

Front. Astron. Space Sci. 5:11. doi: 10.3389/fspas.2018.00011

\section{The Future of Astrometry in Space}

\author{
Antonella Vallenari* \\ Astronomical Observatory of Padova, National Institute for Astrophysics (INAF), Padova, Italy
}

This contribution focuses on the importance of astrometry and on its future developments. Over the centuries astrometry has greatly contributed to the advance of the knowledge of the Universe. Nowadays a major breakthrough is on the way due to astrometric sky surveys from space. ESA space missions Hipparcos first and then Gaia point out the outstanding contribution that space astrometry can provide to our knowledge in many fields of astrophysics, going from the Milky Way formation and evolution, to stellar astrophysics, extra-galactic astrophysics, and fundamental physics. We briefly outline the properties of Gaia first and second data release, and the accuracies expected end-of-mission. The next big advance in space astrometry would be either to improve the astrometric accuracy of one order of magnitude, or to move to a different wavelength domain. While both options have the potential to bring us in a new era of discovery, they have to face enormous issues. We summarize the future directions in space astrometry that are proposed or under investigation by the scientific community, their main challenges and the expected outcome.

Keywords: astrometry, parallaxes, proper motions, space mission, catalogs, sky surveys

\section{INTRODUCTION}

Astrometry is concerned with the measurements of the positions and motions of celestial objects, going from planets and other Solar system objects, through stars within our Galaxy, to, at least in principle, to galaxies and clusters of galaxies within the Universe. The description of the celestial sphere is probably one of the oldest fields of scientific investigation. Since the beginning of the human history, measuring the positions of the celestial objects was of fundamental importance to understand the structure of the visible sky. It had also several practical applications, such as calendars, navigation, and agriculture.

In ancient times, and until about a century ago, astronomers were essentially astrometrists, measuring the effemerides of the stars. One of the first and most famous Catalog of visible stars was compiled by Hipparchus in the 2nd Century BC, and listed no more than 1,000 stars. It remained the reference for a thousand of years. However, the original catalog of Hipparchus did not survive the centuries and most of what is known today originates from the Arabic translators of Ptolemy's 2nd-century Almagest ("The Greatest") which has adopted the entire star catalog of Hipparchus. In 1437, the Ulugh Beg Catalog was completed with a thirty-six meter radius marble sextant in Samarkand and included slightly less than 1,000 stars. A recent comparison with modern measurements from the Hipparcos satellite shows that both catalogs had an accuracy of the order of 22-30 arcmin (Verbunt and van Gent, 2012). Over the centuries, Catalogs with improved accuracy were published. In 1598, due to the substantial advance in the technical possibilities, Tycho Brahe was able to measure positions with uncertainties of about 2 arcmin (Høg, 2017). Hevelius catalog was built about a century later, but it was still of comparable quality. A revolution in the field took place when the telescope was invented and used for astronomical observations (Chapman, 1990). In 1609 Galileo Galilei had the idea to use it to observe the sky. In the Sidereus Nuncius 
published in 1610, Galileo reported about his observations of the Moon, the Jupiter moons called Pallae Medicee to honor the Medici family, and the nebulae, resolved into stars for the first time. His studies supported the heliocentric theory, leading to a conflict with the officials of Catholic Church. Scientists began to understand that a consequence of heliocentric hypothesis and of the gravity theory by Newton (1687) is that the so-called fixed stars could not be considered as having fixed positions in space. Unless they are at infinite distance, they are expected to have a shift in their apparent position, i.e., a parallax motion due to the annual revolution of Earth around the Sun. This idea provoked an increasing interest to detect this shift to prove or disprove this theory. In the late seventeenth and early eighteenth centuries instruments were substantially improved and a transit telescope was in use. Taking advantage of a stable mounting, in the second half of 18th Century, Bradley measured the position of stars within one second of arc. His observations of Gamma Draconis leaded to the discovery of a small systematic shift in the star position, the stellar aberration, announced in 1729 (Chapman, 1990). The first parallax was measured by Bessel in 1838 for the fast moving binary star 61 Cygni (Fricke, 1985). This confirmed that stars were at a finite distance from us, revealing the scale of the Universe (Perryman, 2009). Few years later the parallaxes of Vega and Alpha-Centauri were measured, with a uncertainties of the order of 60-100 mas. Very slowly the number of known parallaxes increased. In Schlesinger (1927) listed 1,800 parallaxes using a new method, based on the use of photographic plates. In 1995 The Yale Catalog included less than 8000 objects with a mean error of 10-20 mas (van Altena et al., 1995). More details can be found in the review by Perryman (2012). A gigantic leap took place with the adoption of space techniques, in the ESA Hipparcos mission first and then in Gaia. Since then, astrometry importance has grown enormously, holding the key not only to understand our Galaxy, but also to make an incredible breakthrough in many field of astrophysics.

Space astrometry objectives are to provide measurements of the positions of the objects with respect to reference stars whose coordinates are known from other means (relative astrometry) or/and to derive positions in a consistent full-sky reference frame (global astrometry). This review focuses on the recent advances on global astrometry and on the future developments and challenges. In section 2 we summarize the Hipparcos mission; in section 3 we present the performances and the recent exciting results of the Gaia mission; in section 4 we discuss the future directions of global astrometry, their expected science outcome and the main challenges they have to face; in section 5 we draw a few concluding remarks.

\section{HIPPARCOS}

Earth atmosphere provides a clear limitation to the accuracy of the parallax measurements. The obvious next step was then to go to space making also use of very stable instruments. The idea was first proposed in the sixties, by Pierre Lacroute (Perryman, 2012). He proposed an instrument able to observe in two separate directions at the same time. These two lines sight were then combined on the same focal plane, providing wide-angle measurements over the entire sky. Hipparcos was then proposed in 1967 and was the first astrometric space mission. The European Space Agency (ESA) approval came in 1980 and finally the satellite was launched on 1989. Hipparcos provided the positions, parallaxes, and annual proper motions for about 100,000 stars with an unprecedented accuracy of 0.7-0.9 milliarcsec (mas) for stars brighter than 9 mag. The first Catalog was produced in 1997 including astrometry and photometry (ESA, 1997). A new reduction with improved accuracy for the bright stars was published by van Leeuwen (2007). Hipparcos improved the astrometry accuracy by a factor of fifty over its predecessors (Perryman et al., 1997). One of the main outcome of the Hipparcos mission was the definition of a precise celestial reference frame in the optical, i.e., a set of reference stars with precise positions and known proper motions across the celestial sphere, linking together observations done at different locations and different times. This reference frame is the optical counterpart of the radio reference frame. It coincides with the International Celestial Reference Frame (ICRF) axes at a level of \pm 0.6 mas and its proper motions are consistent with an inertial system at the level of \pm-0.25 mas/yr (Lindegren and Perryman, 1997b). These data were the reference for astrometry for about 20 years (Perryman, 2009). This mission had an enormous impact on the astrophysics. It is impossible to quote all the papers based on Hipparcos/Tycho-2 data. A detailed review can be found in Perryman (2009). Among the many papers dealing with the properties of the Galactic populations in the solar vicinity, we quote Reddy et al. (2006) and Fuhrmann (1998) for early studies of disk and halo kinematics and metallicity distributions.

Nordström et al. (2004) made use of the parallaxes and proper motions, together with metallicities based on uvby $\beta$ photometry, to derive the ages and Galactic orbits for a complete sample of more than 16,000 FG dwarfs in the solar neighborhood. This Catalog has been widely used in the investigation of the thin/thick disk kinematics and of the relations between kinematics, age, and metallicity in Galactic populations. Very recently the data allow to probe the influence of radial migration on disk evolution (Schönrich and Binney, 2009; Aumer et al., 2017).

Hipparcos astrometry has improved the census of nearby young stellar groups and related star-forming regions (de Zeeuw et al., 1999). It lead to a more precise definition of the evolution of the stars (Pietrinferni et al., 2004; Valenti and Fischer, 2005) and of the properties of stellar aggregates in general (Perryman et al., 1998; Kharchenko et al., 2005) and was also used to characterize stars orbited by planetary mass companions (Santos et al., 2004).

\section{THE GAIA MISSION}

Following the success of Hipparcos, more ambitious astrometric space missions were proposed. Most of these proposals were finally not accepted besides their enormous scientific potential. We recall the German DIVA satellite mission (Bastian et al., 1996), NASA's Space Interferometry Mission SIM, SIM-Planet Quest, FAME (Johnston et al., 2000), and the Russian OSIRIS 
(Bagrov et al., 1999). Finally, the European Space Agency Gaia mission was the only one that was approved. Gaia was originally designed as an astrometric optical interferometer with baseline of a few meters contained in a single payload and operated in a continuously scanning mode. Another main improvement over Hipparcos was the possibility of radial velocities and spectrophotometric measurements by the onboard instrumentation (Lindegren and Perryman, 1997a). The target was to reach the accuracy of $10 \mu$ arcsec or better. Finally, twenty years after Hipparcos, Gaia was approved by ESA in October 2000. The initial optical design was revised and became very similar to Hipparcos, but with enhanced capabilities. The satellite was launched in 2013, with 5 year nominal mission prevision. However, an extension to 10 years of the operations has already been proposed to ESA. At the time of writing, the approval is still pending. The technical description of the spacecraft, of the payload and a summary of the scientific performances are presented in Gaia Collaboration et al. (2016b).

The first data release (Gaia DR1) took place in 2016, the second (Gaia DR2) will be in April 2018, the third is expected in 2020. The final one of the nominal mission is planned after a post-operation processing of all the available data at the end of 2022 and would include astrometry, photometry, radial velocities and information about a large variety of objects, including stars, quasars, galaxies, variables, binaries, solar system objects, exoplanets. Gaia mission will revolutionize astronomy in the coming decades. Gaia science case was first presented in de Boer et al. (2000) and then described in numerous papers. We quote among others Perryman et al. (2001), de Bruijne (2012), Wilkinson et al. (2005), Gaia Collaboration et al. (2016b) and references therein. Here we briefly summarize a few key aspects. One of the main scientific targets of Gaia, if not the principle target, is the Milky Way. Our Galaxy is an impressive laboratory that we can use to reach a detailed understanding of how stars and galaxies form and how they evolve. In turn, stars host planets and our planetary system provides pointers to extra-solar planets. Linking our local environment, the solar vicinity, the disk, the halo, to our Galaxy as a whole, is the key to understand the evolution of the galaxies. Cosmological models predict the formation of large structures through the merging of smaller sub-units. Deciphering the assembly history of our Galaxy is possible through a detailed mapping of the structure, dynamics, chemical composition, and age distribution of its stellar constituents. As an astrometric survey mission, Gaia provides parallaxes, motions in space (proper motions and radial velocities) and astrophysical characterization (through photometry and spectroscopy) for more than one billion stars down to $G=20.7$ mag throughout most of the Galaxy and the Local Group galaxies. The end-of mission (5 years) accuracy is expected to be $25 \mu$-as at $G=15$ and better than $300 \mu$ as at $G=20$. Radial velocities will be known with an accuracy better than $5 \mathrm{~km} / \mathrm{s}$ for GOV stars brighter than $G=15.5(13 \mathrm{~km} / \mathrm{s}$ at $G=16.5$ ) and of $5 \mathrm{~km} / \mathrm{s}$ for a K0V star at $G=16$. Gaia is the cutting edge of Galactic astronomy and will have a major impact across all areas of astronomy and astrophysics, and at all scales. Gaia will allow to study the Galactic structure tracing the disk, the halo and spiral structure, the dark matter content through the detection of halo streams. The difficulty of establishing accurate distances over cosmologically significant scales has made the determination of the expansion rate at the current epoch $\left(H_{0}\right)$ extremely challenging. Deriving the extra-galactic distance scale is a crucial problem of modern astrophysics. Gaia will provide the calibration through direct measurement of parallaxes for the local distance indicators (Cepheids, RR-Lyrae). Gaia will unravel the star formation history of our Galaxy and the kinematics of nearby galaxies. Extra-solar planet detection through astrometry and photometric transit will be possible down to Jupiter-mass objects. In addition fundamental physics tests (relativistic parameters) and stellar physics improvements will be under reach. The challenge will be to built theories and models to reproduce the exquisite details in which our Milky Way is being depicted (Binney, 2017).

\subsection{Gaia First Data Release}

The first Gaia data release is based on the data collected during the first 14 months of the nominal mission. Gaia DR1 properties and limitations are described in Gaia Collaboration et al. (2016a) and references therein. Gaia DR1 supplies the astronomical community with positions, G-band photometry for slightly more than 1 billion objects, and a number of RR-Lyrae and Cepheids light curves. For a subset of objects, about two millions, (the TGAS sample) positions, parallaxes and proper motions are derived using as prior the positions of the Hipparcos and Tycho 2 Catalogs. It should be pointed out that DR1 parallaxes and proper motions are independent from Hipparcos and Tycho-2 data. More information can be found in Lindegren et al. (2016). The typical uncertainties are of the order of about 0.3 mas for the positions and for the parallaxes, and about $1 \mathrm{mas} / \mathrm{yr}$ for the proper motions. Gaia DR1 has defined a new optical reference frame aligned with the ICRF at 0.1 mas (or better) at epoch J2015.0 (Mignard et al., 2016). Gaia G-band magnitudes are given with an impressive accuracy of the order of a few milli-mag for $G<13$ and of $0.03 \mathrm{mag}$ at the faint end. This first data release is already a substantial improvement over the Hipparcos astrometry. However, the quality of the data were limited by the available sky coverage, the still immature calibrations (for instance preliminary modeling of dependence in color and time of the point spread function of the Gaia telescopes, and of the satellite attitude). A systematic term on parallax zero point is still present, i.e., a global offset below 0.1 mas whose value depends on coordinates. Due to the combined effect of the filtering, sky coverage and crowding, the completeness of the Gaia Catalog is a complex function of the position in the sky, of the star density, and of the magnitude. It is also very limited in dense areas on the sky (the Bulge, the globular cluster centers, the low latitude disk Arenou et al., 2017).

Independent verification of Gaia DR1 astrometric quality was performed by the scientific community with a variety of methods. We recall that the comparison with Hubble Space Telescope Fine Guidance Sensor interferometric astrometry (Benedict et al., 2017), with asteroseismic distances (De Ridder et al., 2016; Huber et al., 2017), and with eclipsing binaries (Stassun and Torres, 2016) confirm the presence of a small parallax zero point offset, as already found by Arenou et al. (2017). 
One of the first results of the Gaia DR1 was the solution of the so-called Pleiades distance controversy. The fact that Hipparcos distance to the Pleiades is significantly different from any distance derived with other methods (Melis et al., 2014) has puzzled astronomers for years. Now, the new Gaia parallax reconciles Pleiades distance with literature determinations (Gaia Collaboration et al., 2016a, 2017).

DR1 astrometry and photometry have already become a reference in literature, and have been widely used for calibration (see for instance Magnier and Pan-STARRS Team, 2018 concerning the survey Pan-STARRS, or Nidever et al., 2017 for SMASH). Exciting results on Galactic structure and kinematics were derived combining Gaia astrometry with other photometric or spectroscopic surveys. We quote, among others, Iorio et al. (2018) who derive the first all sky view of the Galactic halo; Helmi et al. (2017) identify a number of substructures in the halo, suggesting that a very high halo fraction was built via accretion. Hunt et al. (2018) studying Hercules stream favor a fast bar model. Massari et al. (2017) derive the 3D motion of the Sculptor putting constraints on its dark matter content. Cantat-Gaudin et al. (2018) obtain the ages and the kinematics of a sample of nearby open clusters. Ward and Kruijssen (2018) analyzing the kinematics of young OB associations, come to the conclusion that not all the stars formed in clusters. TGAS data support the idea that star formation has a hierarchical nature. In this view, largescale associations can form in-situ following the fractal structure of the molecular cloud.

\subsection{Gaia Second Data Release}

The second data release (Gaia DR2) is now approaching. Based on 22 months of data, a full astrometric solution is derived, without imposing any prior. Positions, parallaxes, and proper motions will be available for more than 1.3 billion stars, down to $G=21$. The uncertainties on the parallaxes reach 0.04 mas at $G<15$, and 0.7 mas at $G \sim 20$. In addition six millions of stars brighter than $G \sim 12$ will have radial velocities with a precision of $200-300 \mathrm{~m} / \mathrm{s}$ at the bright end, and of $1.2 \mathrm{~km} / \mathrm{s}$ at the faint end. This is the largest data base of objects having six-parameter solution. More than 1.5 billion objects have $G$, GBP, GRP magnitudes with precision at the milli-mag level at the bright end. In addition Gaia DR2 will contain epoch astrometry for more than 13,000 known asteroids, light curves for more than 500,000 variables. This represents undoubtedly an unprecedented data base for astronomers. Completeness has improved over Gaia DR1. The systematics on the parallax zero point are reduced to the level of about $30 \mu$ as. At the faint end, $G>19$, the GBP and GRP are affected by an imperfect background subtraction. Needless to say that Gaia DR2 will revolutionize our understanding of the Milky Way and beyond. The milli-mag level magnitudes will allow a comparison with stellar models. Star clusters having secure membership derived from DR2 astrometry will provide a test-bed for stellar models allowing further refinements and calibration of effects such as the overshoot and mixing in general in the stellar cores, the rotation, the magnetic fields. This should ultimately lead to a significant improvement in the reliability of those models and their application in various fields, such as providing age estimates for field stars, and stellar population templates for extra-galactic studies. Cluster formation and disruption in different Galactic environments will be within the reach of DR2 data, putting additional constraints on the relation between clusters and field population. Galactic kinematics, halo substructures and Galaxy modeling will benefit from this enormous data-base. Gaia DR2 will clarify how streaming motions affect the Galactic disc, allowing a better definition of the processes involved in galaxy formation and evolution. A recent work has pointed out that the distance uncertainties can create artificial wave-like patterns (Carrillo et al., 2017). Gaia DR2 can probe unexplored regions of the Galaxy, allowing us to put significant constraints on the geometry of the Galactic potential (Posti et al., 2017) and on the accretion history of the halo.

Gaia DR2 and the increasingly richer following releases will undoubtedly provide answers, but may also point to unexpected problems, and to completely new directions in which to take astrophysics.

\section{FUTURE DIRECTIONS OF GLOBAL ASTROMETRY}

The decision of undertaking astrometry from space done with Hipparcos made a true revolution in comparison with groundbased Catalogs. Then Gaia constitutes a jump of two orders of magnitudes in accuracy with respect to Hipparcos. While still awaiting the final Gaia Catalog, the debate in the scientific community is on the future directions of astrometry. In the near future several space missions and facilities will become active. The global astronomical context will be influenced by large ground based surveys such as Pan-STARRS, Lamost, WEAVE, MOONS, and 4MOST. One of the main goals of LSST is to obtain high-quality astrometry, i.e., parallaxes and proper motions. Predicted parallax errors of 0.6 mas at $r=21$ and of about 3mas at $r=24$ are a natural extension at fainter magnitudes of the Gaia parallaxes (Ivezić et al., 2015). New astronomical mega-facilities such as ALMA, E-ELT, SKA, and WFIRST will deliver their data. ESA Euclid space mission will explore the expansion history of the universe and the evolution of cosmic structures by measuring shapes and redshifts of galaxies as well as the distribution of clusters of galaxies over a large fraction of the sky. Clearly the main driver for Euclid is the nature of dark energy, but it goes without saying that Euclid science will cover a vast range of topics, from cosmology to galaxy evolution to planetary research. In this global framework, future directions of global astrometry should provide complementary information. Several options have been envisaged:

- global astrometry in sub- $\mu a$ s or even nano-arcsec regime

- global astrometry in the near-infrared (NIR)

- global astrometry in the optical in a new Gaia-like mission

This review focuses on global astrometry. However, before discussing its prospects in the following sections, we would like to spend a few words on the possibilities offered by relative astrometry. The advantage of this approach is that high relative 
position accuracy can be achieved on small angular scale, allowing the study of higher order positional effects modifying relative positions, as in the case of binary stars or extra-solar planets.

\subsection{Relative Astrometry (Pointed Mission) in the Sub- $\mu$ as Regime}

High precision sub-microarcsec relative astrometry can address many science cases, from the exoplanet detection to the probing of the dark matter. The Hubble Space Telescope has proven to be able to yield sub-milli-arc-second accuracy in differential astrometry (see for instance Libralato et al., 2018). The new generation space telescopes such as WFIRST and JWST can in principle go much further, provided that instrumental issues and systematics such as stability, geometric distortions in the field are understood and accounted for.

Theia was proposed as ESA M-class mission in 2017 (The Theia Collaboration et al., 2017). Its science case well illustrates the possibilities of sub-micro-arcsec relative astrometry. Theia goal is to perform relative astrometry at $0.3 \mu$ as precision for planetary detection and $10 \mu$ as for dark matter studies. Theia can probe the dark matter distribution in dwarf spheroidals, the outer shape of the Milky Way dark matter (DM) halo, and the power spectrum of density perturbations. Dwarf galaxies are dark matter dominated. The shape of the dark matter cores (either large or cuspy) depends on a number of different processes (star formation, self-interaction), (Read et al., 2016). High precision proper motions remove the degeneracy between radial dark matter profile and orbital anisotropy, putting additional constraints on the nature of the dark matter, clarifying whether the DM is warmer than $\Lambda \mathrm{CDM}$ models predict, what is the level of self-interactions, and finally test whether its properties can reduce the number of expected satellites. Concerning exoplanets, differential astrometry provides estimates of the mass and three-dimensional orbital parameters which are fundamental to models of planetary evolution, bio-signature identification, and habitability. This would provide a target data base to be used to search planetary atmospheres for exo-life with the next generation of space telescopes such as James Webb Space Telescope, WFIRST, and E-ELT (Schwieterman et al., 2016). Theia precision can easily be reached with a $0.8 \mathrm{~m}$ three mirrors anastigmatic telescope with a single focal plane, covering a $0.5^{\circ}$ field of view. Relative astrometry can be a very realistic option for future astrometric developments, although then existing or planned facilities can provide a way through.

\subsection{Sub- $\mu$ as Global Astrometry}

Global astrometry at the sub- $\mu$ as level will be the next big breakthrough that will significantly impact on the many fields of astrophysics, cosmology, and gravitation. It will bring astrometry from the local environment to the cosmological scale. While the science case for this is exciting, this option has to face major technological challenges.

\subsubsection{Technological Challenges}

The requirement to have sub- $\mu$ as or nano-arcsec astrometry in the optical is particularly demanding and implies futuristic instrument design. The astrometric precision scales with $1 / D$ where $D$ is the aperture size of the collecting mirror or the interferometer baseline. The sub- $\mu$ as accuracy requirement in the optical translates in an interferometric mission with baseline of 100-1,000 m (Perryman, 2004; Lindegren, 2007). Thus satellites with precision formation flying will be mandatory. Thermo-mechanical stability of the entire spacecraft, attitude control, and knowledge of the barycentric velocity of the spacecraft down to the required level will be really far from trivial and would imply significant technological development (Brown, 2014).

The whole data analysis will be extremely challenging. Indeed the simple treatment of the time dependence of source coordinates now applied to the interpretation of the Gaia data will not be sufficient and new modeling paradigms need to be explored. The effects of the sources of astrometric jitter (star spots, faculae, or micro-lensing in crowded regions) on the interpretation of image locations in the data stream will be relevant and requires further research. In addition we are still lacking of suitable modeling to correct astrometric measurements for relativistic effects at the nano-arcsec level (Klioner, 2003). This requires a refinement of the currently employed models and also a substantially improved knowledge of the solar system (e.g., asteroid masses, Kopeikin and Gwinn, 2000).

\subsubsection{Science Case: Real Time Cosmology}

The importance of sub- $\mu$ as astrometry in the framework of stellar populations, Local Group galaxies, stellar physics and exoplanets has been extensively described by Perryman (2004) and by Unwin et al. (2008) in the context of the SIM mission. Here we focus on two other science cases that will open a new window, i.e., real time cosmology and gravitational wave detection.

One of most exciting applications of the astrometry stays in a new field of study, real-time cosmology (Brown, 2014). The main point is that while most cosmological observations have a geometric nature (see for instance the use of standard candles to measure distances and the expansion of the Universe), in real time cosmology the measurements involve dynamics, and are independent from cosmological models. This was already noticed in de Boer et al. (2000). Real-time cosmology measures variations of radial and transverse position and/or velocity of a given source at cosmological scale. The red-shift is the first well known real time observable. The transverse motion of external galaxies and quasars is the analogous in the transverse direction. The astrometry of quasars is affected by several effects, first of all the intrinsic proper motion due to relativistic jets and the secular aberration drift due to the acceleration of the Sun around the Galactic center. The secular aberration drift cannot be distinguished from other effects, like cosmic parallaxes. However, if we assume we can model it as a dipole, we get an amplitude of the order of 4.9-6 $\mu \mathrm{as} / y r$ (Truebenbach and Darling, 2017). Then, correlated proper motions of quasars can be used to probe cosmological models, for instance obtaining geometrical distances independent of the cosmological distance ladders, testing the Universe isotropic expansion, measuring the collapse of large scale structures, and, finally providing estimates of the 
upper limit of the energy flux of the stochastic gravitational wave background. To do this one needs to assume that all systematics in higher order harmonics come from the gravitational wave background. The quadrupole component and higher order harmonics should be sensitive to Hubble constant anisotropy or to primordial gravitational waves of long wavelengths (Titov et al., 2011; Mignard and Klioner, 2012; Truebenbach and Darling, 2017). Bachchan et al. (2016) summarize the known astrometric effects related to cosmology. In the following, we briefly comment on these items.

An homogeneous and isotropic metric (the FriedmannRobertson-Walker metric) is one of the main assumption behind our standard cosmological models. However this is still an open issue. A deviation from homogeneity and isotropy is proposed as an alternative view to explain the accelerated expansion of the Universe without a dark energy field. Any anisotropy in the expansion of the Universe would produce a variation in time of the angular separation between two sources, called cosmic parallaxes (Quercellini et al., 2009). This effect is a function of the red-shift. In an isotropic expansion, the cosmic parallax vanishes, since the angular separations between sources is expected to be constant (but for the peculiar motions). Quercellini et al. (2009) estimate that, depending on which scenario is considered, either Bianchi 1 (intrinsically anisotropic expansion due to anisotropically distributed dark energy density seen by a central observer), or Lemaitre-Tolman-Bondi void model (universe inhomogeneous and isotropic with an off-center observer), the expected proper motion pattern is of the order of $0.2 \mu \mathrm{as} / \mathrm{yr}$, or $0.02 \mu a s / y r$. A positive detection of large-scale cosmic parallax would disprove one of the main hypotheses of modern cosmology, isotropy.

Astrometry can measure the collapse of structures. Small separation objects that are in gravitationally interacting systems (for instance filaments) are expected to present a net decrease in their angular separations while collapsing. Unbound objects just moving with the Hubble flow will show no net angular separation changes. Most recent determinations using VLBI quasar Catalog, put an upper limit on the rate of convergence of large-scale structure of $-11.4 \mu a s / y r$ for extra-galactic objects within 100 co-moving Mpc of each other (Truebenbach and Darling, 2018).

Finally, due the motion of the Earth/Sun system with respect to the cosmic microwave background, we have a basis of $80 \mathrm{au} / \mathrm{yr}$ on which parallactic effects can be measured as quasar proper motions over a sufficiently long time period (at least 10 years). This effect depends on the redshift and on the fundamental cosmological parameters. At very low redshift $(z \sim 0.01)$ it is of the order of 1-2 $\mu a s / y r$ (Kardashev, 1986), and can possibly be within reach of Gaia end-of mission uncertainties. This shift would lead to geometrical constraints on the dark energy with a significant figure of merit (Ding and Croft, 2009).

As we have discussed, all these measurements present considerable technical challenges and require an enormous control of both systematic and statistical errors. With a few exceptions, they are far beyond the possibilities of Gaia (Ding and Croft, 2009), and even combining present measurements with a new Gaia mission (either in the optical or in the infrared, see below) would only provide very limited constraints (Hobbs et al., 2016).

\subsubsection{Science Case: Gravitational Waves Detection}

The detection of gravitational waves by the Advanced LIGO experiment has just opened the way to new explorations (Abbott et al., 2016). However lower frequencies are inaccessible to ground-based instruments. Further progress is expected from the space-based detector, LISA. The possibility of using astrometry to detect Gravitational waves was proposed by many authors (see for instance Braginsky et al., 1990; de Boer et al., 2000). The passage of a gravitational wave provokes a deflection of the apparent position of the star, i.e., an apparent proper motion. The astrometric deflection depends on a telescope term and on a star term. This second term will be different from each star and definitely smaller for distant sources, while the telescope term is common. This implies that the motions are coherent across the sky. Thus, repeated astrometric measurements across the whole sky would allow the identification of the deflection pattern due to a gravitational wave passage.

One should distinguish the regime of ultra-low frequency gravitational waves that directly influence apparent proper motions (Pyne and Carroll, 1996; Gwinn et al., 1997; Book and Flanagan, 2011) and the regime of higher-frequency gravitation waves, the effects of which remain in the residuals of the astrometric solution (Klioner, 2018). In the regime of the ultra-low frequency primordial stochastic gravitational wave background, Gaia is expected to set an upper limit to the energy of the gravitational waves with frequencies $v<6.410^{-9}$ $\mathrm{Hz}$ at the level of $\Omega_{G W}<0.00012 h_{1} 00^{-2}$, where $h_{1} 00=$ $H /(100 \mathrm{Km} / \mathrm{s} / \mathrm{Mpc})$ and $\Omega_{G W}$ is the ratio of the energy density in gravitational waves to the energy density needed to close the Universe. This is a significant improvement over the best VLBI results (Mignard and Klioner, 2012; Klioner, 2018). These frequencies correspond to the stochastic background produced by the superposition many monochromatic signals, or of primordial origin.

Recent papers have explored the astrometric detection of gravitational waves of higher frequencies in the context of Gaialike astrometric missions (Moore et al., 2017; Klioner, 2018). Assuming that all the signals with period less than 2 Gaia rotational periods will not detectable and that the period of the detectable waves should be considerably shorter than the length of the mission, one comes to the conclusion that Gaia is sensitive to wavelengths in the range $6.4 \times 10^{-9} \mathrm{~Hz}<v<$ $3 \times 10^{-5} \mathrm{~Hz}$ (Klioner, 2018). A detailed practical algorithm to search the gravitational wave signatures in the residuals of Gaia-like astrometric solution is formulated in Klioner (2018). In this frequency range Gaia can search for signals of e.g., binary supermassive black holes. In general, prospects for astrometric detection are not very encouraging (Schutz, 2009), but nevertheless gravitational wave sources detectable by Gaia still may exist.

What are the implications for a future nano-as mission? The astrometric effect due to a stochastic gravitational wave background on a single object proper motion is expected to be less than 0.1 $\mu \mathrm{as} / \mathrm{yr}$ from Big Bang nucleosynthesis constraints, 
but millisecond pulsar timing suggests that it is at nano-as/yr level (Book and Flanagan, 2011). A future mission at the nano-as level has fair chances to make a detection. A few million sources with such accurate proper motions (but little or no systematics) would allow the detection of stochastic background down to the level of $\Omega_{G W} \sim 10^{-14}$ (de Boer et al., 2000).

\subsection{Infrared Astrometric Missions}

Gaia is operating at optical wavelength. The very obscured regions of our Galaxy, i.e., the central bulge, the inner disk, the star forming regions and the spiral arms cannot be explored. A natural evolution would be to switch to astrometry in the infrared. Near-field cosmology is of fundamental importance, since detailed studies of the Galaxy underlie our understanding of universal processes over cosmic time. It has become clear in the recent past that one component of the Milky Way cannot be separated from another (Bland-Hawthorn and Gerhard, 2016). Migration affects thin/thick disk formation (Sellwood and Binney, 2002; Aumer et al., 2017). Disk instability might be responsible for the bar formation (see among others Athanassoula et al., 2017). The inner Galaxy is still puzzling. The bulge is formed by a mixture of populations having different orbits, but is unclear how many we have (Ness et al., 2013). It is still under discussion if a classical bulge formed by early mergers is present or if the old-metal poor population traced by the RRLyrae is indeed related to the halo (Pietrukowicz et al., 2015; Pérez-Villegas et al., 2017). Finally we do not know how nuclear disk, inner disk and bar are connected (Ness et al., 2013) The outer bulge/halo transition needs to be explored; the dark matter distribution in the inner Galaxy is unknown (see Gerhard, 2017 and references therein). In this context, it is important to have a global view of the kinematics and dynamics of all the Galaxy components. New astrometric missions in the NIR are already under study to cover the gap and allow a better understanding of the our Galaxy as a whole. An ESA mission, Gaia-NIR, extending Gaia capabilities in the infrared after 20 years has been proposed (Hobbs et al., 2016). The goal is to discuss the above issues with an accuracy comparable to Gaia in the obscured regions of the Galaxy. Gaia and Gaia-NIR measurements can be combined producing higher accuracy astrometry in the whole Galaxy (see following section). The challenge of this astrometric mission is related to the use of infrared detectors in time delay integration (TDI) mode, for which technology is not yet mature. $\mathrm{HgCdTe}$ detectors covering a range between 400 and 2,500 nm are proposed. The problem is the high read-out noise of these detectors. High quality astrometry with performances similar to Gaia requires a low read-out noise, of the order of $10 \mathrm{e}^{-}$. At the current status, this level can be reached in near-infrared detectors per pixel. However, this would lead to an extremely high read-out noise when the detectors are used in TDI mode. Alternatively, a limits at 1,600 nm is a fall back option that would still allow to meet the scientific goals. This option implies optical CCDs covering the range from 400 to $1,600 \mathrm{~nm}$, and NIR detectors in a separated field.

We should also recall the Japan Aerospace Exploration Agency space missions Small- Jasmine and Jasmine (Yamada et al., 2013; Gouda, 2015), currently under development. They are meant to target a small part of the inner Galaxy, close to the Galactic center and the whole bulge respectively with an accuracy of $10 \mu a s$ for stars brighter than $\mathrm{H}_{w} \sim 11.5$. The scientific objectives are to study the dynamics of the Galactic center and of the bulge, and clarify the nature of the super-massive black hole. The NanoJASMINE micro-satellite project is meant to be a prototype for Small-JASMINE and is expected to conduct an whole sky survey with accuracy of 3 mas at a magnitude $\mathrm{z}_{w} \sim 7.5$.

\subsection{Repeating the Gaia Mission}

Another option proposed to ESA is to have a new Gaia mission in about 20 years from now (Høg, 2014). To repeat a mission is not usually done, but here it would have the advantage of a very realistic possibility, built on the knowledge of Gaia. Two five (or ten-year) Gaia-like missions with assumed accuracy of $25 \mu a s / y$ separated by 20 years would result in an improvement of 14 (or 20) times on proper motions for more than one billion stars and in more accurate parallaxes. The accuracy on proper motion will be of the order of $1.77 \mu a s / y r$ (5 year option). While Gaia at its 5 -years nominal duration provides high precision proper motion mapping of the streams in the halo up to $20 \mathrm{Kpc}$ (or $30 \mathrm{Kpc}$ in 10 years), with a new mission stellar streams in the halo out to 100 $\mathrm{Kpc}$ can be detected providing fundamental information about galaxy formation process. Indeed recent studies have pointed out that a transition in halo properties (metallicity, density distribution, degree of lumpiness) between the inner and outer regions takes place at farther distances. This interface could also be related to the in-situ vs. the accreted halo. Young stellar debris, accreted less than $8 \mathrm{Gyr}$ ago are expected to be more easily found at larger distances. High precision kinematics of gaps in the streams can be used to trace the dark matter content of our Galaxy (Helmi and Koppelman, 2016). Several other science cases can be addressed by these observations going from exoplanets, to brown dwarfs, and binaries. Internal proper motions of Local Group galaxies can be resolved, allowing determination of the dark matter content. This sample will include SPhs and Ultrafaint galaxies that could be the building blocks of our Galaxy. Ultimately this can help understanding the missing satellite problem and the too big to fail problem.

Finally, Gaia optical reference frame will be degraded by about 40 mas over 50 years, as an effect of the cosmic acceleration (or galactic aberration) acting like a systematic proper motion field for the QSOs. With a new mission, the reference frame degradation will be slow down by the same factor affecting the proper motions (Mignard et al., 2016).

\section{CONCLUSIONS}

We are now in the golden age of space astrometry. Gaia exquisite capabilities have established the European science and industry as leaders in high precision astrometry. Gaia science exploitation is just at the beginning, but will likely continue in the coming decades and will drive the development of next major steps in astrophysics. Potential key science drivers and development pathways will become clearer after this process and it cannot be excluded that astrophysics might take unexpected directions. The aim of next generation astrometric missions 
at the $s u b$ - $\mu$ as precision level can be very ambitious: they have the potential to yield the most direct constraints on cosmological models, and on dark energy parameters, but also they can provide limits on the gravitational wave background. Moving to astrometry in the NIR would provide a more complete picture of our Milky Way, and of the Local Group bridging the gaps of Gaia measurements. However both options will require to overcome enormous technical and scientific challenges.

\section{AUTHOR CONTRIBUTIONS}

$\mathrm{AV}$ is responsible of the conception and design of the work. The Author is Deputy Chair of Gaia Science Team and Gaia Data Processing and Analysis Consortium and actively contributed to the development of several of the projects reviewed here. The manuscripts submitted to Frontiers was not previously published or be under consideration for publication elsewhere, neither in whole nor in part. The Author agrees to be accountable for the content of the work.

\section{REFERENCES}

Abbott, B. P., Abbott, R., Abbott, T. D., Abernathy, M. R., Acernese, F., Ackley, K., et al. (2016). Observation of Gravitational Waves from a Binary Black Hole Merger. Phys. Rev. Lett. 116:061102. doi: 10.1103/PhysRevLett.116.061102

Arenou, F., Luri, X., Babusiaux, C., Fabricius, C., Helmi, A., Robin, A. C., et al. (2017). Gaia Data Release 1. Catalogue validation. Astron. Astrophys. 599:A50. doi: 10.1051/0004-6361/201629895

Athanassoula, E., Rodionov, S. A., and Prantzos, N. (2017). Metallicity-dependent kinematics and morphology of the Milky Way bulge. Month. Not. R. Astron. Soc. 467, L46-L50. doi: 10.1093/mnrasl/slw255

Aumer, M., Binney, J., and Schönrich, R. (2017). Migration and kinematics in growing disc galaxies with thin and thick discs. Month. Not. R. Astron. Soc. 470, 3685-3706. doi: 10.1093/mnras/stx1483

Bachchan, R. K., Hobbs, D., and Lindegren, L. (2016). Gaia reference frame amid quasar variability and proper motion patterns in the data. Astron. Astrophys. 589:A71. doi: 10.1051/0004-6361/201527935

Bagrov, A. V., Boyarchuk, A. A., Barabanov, S. I., Mikisha, A. M., Rykhlova, L. V., and Smirnov, M. A. (1999). "OSIRIS: An Optical Stellar Interferometer for Russian Investigations in Space," in Working on the Fringe: Optical and IR Interferometry from Ground and Space (San Francisco, CA), Vol. 194 of Astronomical Society of the Pacific Conference Series, 381.

Bastian, U., Röser, S., Hoeg, E., Mandel, H., Seifert, W., Wagner, S., et al. (1996). DIVA - an interferometric minisatellite for astrometry and photometry. Astronomische Nachr. 317, 281-288. doi: 10.1002/asna.2113170407

Benedict, G. F., McArthur, B. E., Nelan, E. P., and Harrison, T. E. (2017). Astrometry with hubble space telescope fine guidance sensors - a review. Public. Astron. Soc. Pacific 129:12001. doi: 10.1088/1538-3873/129/971/012001

Binney, J. (2017). Self-consistent modelling of our Galaxy with Gaia data. ArXiv e-prints.

Bland-Hawthorn, J., and Gerhard, O. (2016). The galaxy in context: structural, kinematic, and integrated properties. Ann. Rev. Astron. Astrophys. 54, 529-596. doi: 10.1146/annurev-astro-081915-023441

Book, L. G., and Flanagan, É. É. (2011). Astrometric effects of a stochastic gravitational wave background. Phys. Rev. D 83:024024. doi: 10.1103/PhysRevD.83.024024

Braginsky, V. B., Kardashev, N. S., Polnarev, A. G., and Novikov, I. D. (1990). Propagation of electromagnetic radiation in a random field of gravitational waves and space radio interferometry. Nuovo Cimento B Serie 105, 1141-1158.

Brown, A. G. A. (2014). "Space astrometry in the Gaia era and beyond," in EAS Publications Series, eds N. Walton, F. Figueras, L. Balaguer-Nunos, and C. Soubiran, (Les Ulis: EDP Science), Vol. 67, 307-313. doi: 10.1051/eas/1567056

\section{FUNDING}

Funding for the Gaia Data Processing and Analysis Consortium has been provided by national institutions, in particular the institutions participating to the multi-lateral agreement. In particular, the author acknowledges INAF support, and ASI contract to INAF 2014-025-R.I.2015 (Gaia Mission Italian Participation to DPAC).

\section{ACKNOWLEDGMENTS}

This work has made use of the results of the ESA space mission Gaia, the data from which where processed by the Gaia Data Processing and Analysis Consortium (DPAC). Many thanks are due to the members of the Gaia Science Team and Gaia Data Processing and Analysis Consortium for their contribution to the development of the projects reviewed here. The Author is indebted to Sergei Klioner, for many useful discussions about astrometric effects related to cosmology and gravitational waves and a careful reading of the text.

Cantat-Gaudin, T., Vallenari, A., Sordo, R., Pensabene, F., Krone-Martins, A., Moitinho, A., et al. (2018). Characterising Open Clusters in the solar neigbourhood with the Tycho-Gaia Astrometric Solution. ArXiv e-prints.

Carrillo, I., Minchev, I., Kordopatis, G., Steinmetz, M., Binney, J., Anders, F., et al. (2017). Is the Milky Way still breathing? RAVE-Gaia streaming motions. ArXiv e-prints.

Chapman, A. (1990). Book-Review - Dividing the Circle - the Development of Critical Angular Measurement in Astronomy 1500-1850. J. Br. Astron. Assoc. 100:316.

de Boer, K., Gilmore, G., Hoeg, E., Lattanzi, M. G., Lindegren, L., Luri, X., et al. (2000). GAIA Concept and Technology Study Report (The Red Book. Paris: ESA.

de Bruijne, J. H. J. (2012). Science performance of Gaia, ESA's spaceastrometry mission. Astrophys. Space Sci. 341, 31-41. doi: 10.1007/s10509-0121019-4

De Ridder, J., Molenberghs, G., Eyer, L., and Aerts, C. (2016). Asteroseismic versus Gaia distances: a first comparison. Astron. Astrophys. 595:L3. doi: 10.1051/0004-6361/201629799

de Zeeuw, P. T., Hoogerwerf, R., de Bruijne, J. H. J., Brown, A. G. A., and Blaauw, A. (1999). A HIPPARCOS census of the nearby OB associations. Astron. J. 117, 354-399. doi: 10.1086/300682

Ding, F., and Croft, R. A. C. (2009). Future dark energy constraints from measurements of quasar parallax: Gaia, SIM and beyond. Month. Notices R. Astron. Soc. 397, 1739-1747. doi: 10.1111/j.1365-2966.2009.15111.x

ESA ed (1997). The HIPPARCOS and TYCHO Catalogues. Astrometric and Photometric Star Catalogues Derived from the ESA HIPPARCOS Space Astrometry Mission, Vol. 1200. ESA Special Publication.

Fricke, W. (1985). Friedrich Wilhelm Bessel (1784-1846). In Honor of the 200th Anniversary of Bessel's Birth. Astrophys. Space Sci. 110, 11-19. doi: 10.1007/BF00660603

Fuhrmann, K. (1998). Nearby stars of the Galactic disk and halo. Astron. Astrophys. 338, 161-183.

Gaia Collaboration, Brown, A. G. A., Vallenari, A., Prusti, T., de Bruijne, J. H. J., Mignard, F., et al. (2016a). Gaia Data Release 1. Summary of the astrometric, photometric, and survey properties. Astron. Astrophys. 595:A2. doi: 10.1051/0004-6361/201629512

Gaia Collaboration, Prusti, T., de Bruijne, J. H. J., Brown, A. G. A., Vallenari, A., Babusiaux, C., et al. (2016b). The Gaia mission. Astron. Astrophys. 595:A1. doi: 10.1051/0004-6361/201629272

Gaia Collaboration, van Leeuwen, F., Vallenari, A., Jordi, C., Lindegren, L., Bastian, U., et al. (2017). Gaia Data Release 1. Open cluster astrometry: performance, limitations, and future prospects. Astron. Astrophys. 601:A19. doi: 10.1051/0004-6361/201730552 
Gerhard, O. (2017). The barred inner Milky Way: dynamical models from surveys. ArXiv e-prints.

Gouda, N. (2015). Infrared Sapce Astrometry missions: JASMINE. IAU Gen. Assemb. 22:2247720.

Gwinn, C. R., Eubanks, T. M., Pyne, T., Birkinshaw, M., and Matsakis, D. N. (1997). Quasar proper motions and low-frequency gravitational aves. Astrophys. J. 485, 87-91. doi: $10.1086 / 304424$

Helmi, A., and Koppelman, H. H. (2016). The time volution of gaps in Tidal Streams. Astrophys. J. Lett. 828:L10. doi: 10.3847/2041-8205/828/1/L10

Helmi, A., Veljanoski, J., Breddels, M. A., Tian, H., and Sales, L. V. (2017). A box full of chocolates: The rich structure of the nearby stellar halo revealed by Gaia and RAVE. Astron. Astrophys. 598:A58. doi: 10.1051/0004-6361/201629990

Hobbs, D., Høg, E., Mora, A., Crowley, C., McMillan, P., Ranalli, P., et al. (2016). GaiaNIR: Combining optical and Near-Infra-Red (NIR) capabilities with TimeDelay-Integration (TDI) sensors for a future Gaia-like mission. ArXiv e-prints.

Høg, E. (2014). Absolute astrometry in the next 50 years. ArXiv e-prints.

Høg, E. (2017). Selected astrometric catalogues. ArXiv e-prints.

Huber, D., Zinn, J., Bojsen-Hansen, M., Pinsonneault, M., Sahlholdt, C., Serenelli, A., et al. (2017). Asteroseismology and Gaia: testing scaling relations using 2200 kepler stars with TGAS parallaxes. Astrophys. J. 844:102. doi: 10.3847/1538-4357/aa75ca

Hunt, J. A. S., Bovy, J., Pérez-Villegas, A., Holtzman, J. A., Sobeck, J., Chojnowski, D., et al. (2018). The Hercules stream as seen by APOGEE-2 South. Month. Notic. R. Astron. Soc. 474, 95-101. doi: 10.1093/mnras/stx2777

Iorio, G., Belokurov, V., Erkal, D., Koposov, S. E., Nipoti, C., and Fraternali, F. (2018). The first all-sky view of the Milky Way stellar halo with Gaia+2MASS RR Lyrae. Month. Notic. R. Astron. Soc. 474, 2142-2166. doi: 10.1093/mnras/stx2819

Ivezić, Ž., Kahn, S. M., and Eliason, P. (2015). The Gaia-LSST synergy. ArXiv e-prints.

Johnston, K., Gaume, R., Harris, F., Monet, D., Murison, M., Seidelmann, P. K., et al. (2000). "Full-sky Astrometric Mapping Explorer (FAME)," in American Astronomical Society Meeting Abstracts (Washington, DC), Vol. 32 of Bulletin of the American Astronomical Society, 1425.

Kardashev, N. S. (1986). Cosmological proper motion. Astronomicheskii Zhurnal $63,845-849$.

Kharchenko, N. V., Piskunov, A. E., Röser, S., Schilbach, E., and Scholz, R.-D. (2005). Astrophysical parameters of Galactic open clusters. Astron. Astrophys. 438, 1163-1173. doi: 10.1051/0004-6361:20042523

Klioner, S. A. (2003). A practical elativistic model for microarcsecond astrometry in pace. Astron. J. 125, 1580-1597. doi: 10.1086/367593

Klioner, S. A. (2018). Gaia-like astrometry and gravitational waves. Classical Quantum Gravity 35:045005. doi: 10.1088/1361-6382/aa9f57

Kopeikin, S., and Gwinn, C. (2000). "Sub-microarcsecond astrometry and new horizons in relativistic gravitational physics," in IAU Colloq. 180: Towards Models and Constants for Sub-Microarcsecond Astrometry, eds K. J. Johnston, D. D. McCarthy, B. J. Luzum, and G. H. Kaplan (Washington, DC: US Naval Observatory), 303.

Libralato, M., Bellini, A., Bedin, L. R., Moreno D., E., Fernández-Trincado, J. G., Pichardo, B., et al. (2018). The HST Large Programme on $\omega$ Centauri. III. Absolute Proper Motion. Astrophys. J. 854:45. doi: 10.3847/1538-4357/aaa59e

Lindegren, L. (2007). Scientific potential of the future space astrometric missions. Highlights Astron. 14, 481-481. doi: 10.1017/S1743921307011556

Lindegren, L., Lammers, U., Bastian, U., Hernández, J., Klioner, S., Hobbs, D., et al. (2016). Gaia Data Release 1. Astrometry: one billion positions, two million proper motions and parallaxes. Astron. Astrophys. 595:A4. doi: 10.1051/0004-6361/201628714

Lindegren, L., and Perryman, M. A. C. (1997a). "GAIA: Global Astrometric Interferometer for Astrophysics," in Hipparcos - Venice '97, Vol. 402, eds R. M. Bonnet, E., Høg, P. L. Bernacca, L. Emiliani, A. Blaauw, C. Turon, J. Kovalevsky, L. Lindegren, H. Hassan, M. Bouffard, B. Strim, D. Heger, M. A. C. Perryman, and L. Woltjer (ESA Special Publication), 799-802.

Lindegren, L., and Perryman, M. A. C. (1997b). "Optical counterpart of ICRF: HIPPARCOS," in IAU Joint Discussion, ed J. Andersen (Dordrecht: Kluwer Academic Publishers), Vol. 7:21.

Magnier, E. A., and Pan-STARRS Team (2018). "Precision Photometry and Astrometry from Pan-STARRS," in American Astronomical Society Meeting
Abstracts \#231, Vol. 231 of American Astronomical Society Meeting Abstracts, $\# 102.03$.

Massari, D., Breddels, M. A., Helmi, A., Posti, L., Brown, A. G. A., and Tolstoy, E. (2017). 3D motions in the Sculptor dwarf galaxy as a glimpse of a new era. ArXiv e-prints.

Melis, C., Reid, M. J., Mioduszewski, A. J., Stauffer, J. R., and Bower, G. C. (2014). A VLBI resolution of the Pleiades distance controversy. Science 345, 1029-1032. doi: $10.1126 /$ science. 1256101

Mignard, F., and Klioner, S. (2012). Analysis of astrometric catalogues with vector spherical harmonics. Astron. Astrophys. 547:A59. doi: 10.1051/0004-6361/201219927

Mignard, F., Klioner, S., Lindegren, L., Bastian, U., Bombrun, A., Hernández, J., et al. (2016). Gaia Data Release 1. Reference frame and optical properties of ICRF sources. Astron. Astrophys. 595:A5. doi: 10.1051/0004-6361/201629534

Moore, C. J., Mihaylov, D. P., Lasenby, A., and Gilmore, G. (2017). Astrometric Search Method for Individually Resolvable Gravitational Wave Sources with Gaia. Phys. Rev. Lett. 119:261102. doi: 10.1103/PhysRevLett.119.261102

Ness, M., Freeman, K., Athanassoula, E., Wylie-de-Boer, E., Bland-Hawthorn, J., Asplund, M., et al. (2013). ARGOS - III. Stellar populations in the Galactic bulge of the Milky Way. Month. Notic. R. Astron. Soc. 430, 836-857. doi: 10.1093/mnras/sts629

Nidever, D. L., Olsen, K., Walker, A. R., Vivas, A. K., Blum, R. D., Kaleida, C., et al. (2017). SMASH: Survey of the MAgellanic Stellar History. Astron. J. 154:199. doi: 10.3847/1538-3881/aa8d1c

Nordström, B., Mayor, M., Andersen, J., Holmberg, J., Pont, F., Jørgensen, B. R., et al. (2004). The Geneva-Copenhagen survey of the Solar neighbourhood. Ages, metallicities, and kinematic properties of $\sim 14000 \mathrm{~F}$ and $\mathrm{G}$ dwarfs. Astron. Astrophys. 418, 989-1019. doi: 10.1051/0004-6361:20035959

Pérez-Villegas, A., Portail, M., and Gerhard, O. (2017). The stellar halo in the inner Milky Way: predicted shape and kinematics. Month. Notic. R. Astron. Soc. 464, L80-L84. doi: 10.1093/mnrasl/slw189

Perryman, M. (2009). Astronomical Applications of Astrometry: Ten Years of Exploitation of the Hipparcos Satellite Data. Cambridge: Cambridge University Press.

Perryman, M. (2012). The history of astrometry. Euro. Phys. J. H 37, 745-792. doi: 10.1140/epjh/e2012-30039-4

Perryman, M. A. C. (2004). "Detection and Characterization of Extra-Solar Planets: Future Space Missions," in The Search for Other Worlds, Vol. 713, American Institute of Physics Conference Series, eds S. S. Holt and D. Deming (Melville, WA; New York, NY: AIP Publishing), 283-292.

Perryman, M. A. C., Brown, A. G. A., Lebreton, Y., Gomez, A., Turon, C., Cayrel de Strobel, G., et al. (1998). The Hyades: distance, structure, dynamics, and age. Astron. Astrophys. 331, 81-120.

Perryman, M. A. C., de Boer, K. S., Gilmore, G., Høg, E., Lattanzi, M. G., Lindegren, L., et al. (2001). GAIA: Composition, formation and evolution of the Galaxy. Astron. Astrophys. 369, 339-363. doi: 10.1051/0004-6361:20010085

Perryman, M. A. C., Lindegren, L., Kovalevsky, J., Hoeg, E., Bastian, U., Bernacca, P. L., et al. (1997). The HIPPARCOS Catalogue. Astron. Astrophys. 323, L49-L52.

Pietrinferni, A., Cassisi, S., Salaris, M., and Castelli, F. (2004). A large stellar evolution database for population Synthesis Studies. I. Scaled solar models and isochrones. Astrophys. J. 612, 168-190. doi: 10.1086/422498

Pietrukowicz, P., Kozłowski, S., Skowron, J., Soszyński, I., Udalski, A., Poleski, R., et al. (2015). Deciphering the 3D Structure of the Old Galactic Bulge from the OGLE RR Lyrae Stars. Astrophys. J. 811:113. doi: 10.1088/0004-637X/811/2/113

Posti, L., Helmi, A., Veljanoski, J., and Breddels, M. (2017). The dynamically selected stellar halo of the Galaxy with Gaia and the tilt of the velocity ellipsoid. ArXiv e-prints.

Pyne, T., and Carroll, S. M. (1996). Higher-order gravitational perturbations of the cosmic microwave background. Phys. Rev. D 53, 2920-2929. doi: 10.1103/PhysRevD.53.2920

Quercellini, C., Quartin, M., and Amendola, L. (2009). Possibility of detecting anisotropic expansion of the universe by very accurate astrometry measurements. Phys. Rev. Lett. 102:151302. doi: 10.1103/ PhysRevLett.102.151302

Read, J. I., Agertz, O., and Collins, M. L. M. (2016). Dark matter cores all the way down. Month. Notic. R. Astron. Soc. 459, 2573-2590. doi: 10.1093/mnras/ stw713 
Reddy, B. E., Lambert, D. L., and Allende Prieto, C. (2006). Elemental abundance survey of the Galactic thick disc. Month. Notic. R. Astron. Soc. 367, 1329-1366. doi: 10.1111/j.1365-2966.2006.10148.x

Santos, N. C., Israelian, G., and Mayor, M. (2004). Spectroscopic [Fe/H] for 98 extra-solar planet-host stars. Exploring the probability of planet formation. Astron. Astrophys. 415, 1153-1166. doi: 10.1051/0004-6361:20034469

Schlesinger, F. (1927). Photography, astronomical, of precision, Some aspects of (George Darwin Lecture, 1927). Month. Notic. R. Astron. Soc. 87, 506-523. doi: $10.1093 / \mathrm{mnras} / 87.7 .506$

Schönrich, R., and Binney, J. (2009). Chemical evolution with radial mixing. Month. Notic. R. Astron. Soc. 396, 203-222. doi: 10.1111/j.1365-2966. 2009.14750.x

Schutz, B. F. (2009). "Astrometric and timing effects of gravitational waves," in IAU Symposium \#261, American Astronomical Society (Washington, DC), Vol. 261:888.

Schwieterman, E. W., Meadows, V. S., Domagal-Goldman, S. D., Deming, D., Arney, G. N., Luger, R., et al. (2016). Identifying Planetary Biosignature Impostors: Spectral Features of $\mathrm{CO}$ and $\mathrm{O}_{4}$ Resulting from Abiotic $\mathrm{O}_{2} / \mathrm{O}_{3}$ Production. Astrophys. J. Lett. 819:L13. doi: 10.3847/2041-8205/ $819 / 1 / \mathrm{L} 13$

Sellwood, J. A., and Binney, J. J. (2002). Radial mixing in galactic discs. Month. Notic. R. Astron. Soc. 336, 785-796. doi: 10.1046/j.1365-8711.2002.05806.x

Stassun, K. G., and Torres, G. (2016). Evidence for a Systematic Offset of -0.25 mas in the Gaia DR1 Parallaxes. Astrophys. J. Lett. 831:L6. doi: $10.3847 / 2041-8205 / 831 / 1 /$ L6

The Theia Collaboration, Boehm, C., Krone-Martins, A., Amorim, A., AngladaEscude, G., Brandeker, A., et al. (2017). Theia: Faint objects in motion or the new astrometry frontier. ArXiv e-prints.

Titov, O., Lambert, S. B., and Gontier, A.-M. (2011). VLBI measurement of the secular aberration drift. Astron. Astrophys. 529:A91. doi: 10.1051/0004-6361/201015718

Truebenbach, A., and Darling, J. (2018). "“Real-Time" cosmology with extragalactic proper motions: the secular aberration drift and evolution of large-scale structure," in American Astronomical Society Meeting Abstracts \#231, volume 231 of American Astronomical Society Meeting Abstracts, $\# 430.03$.

Truebenbach, A. E., and Darling, J. (2017). The VLBA extragalactic proper motion catalog and a measurement of the secular aberration drift. Astrophys. J. Suppl. 233:3. doi: 10.3847/1538-4365/aa9026
Unwin, S. C., Shao, M., and Edberg, S. J. (2008). "Precision astrometry with a space-based interferometer," in Optical and Infrared Interferometry, Vol. 7013 of procspie, $70132 \mathrm{~L}$.

Valenti, J. A., and Fischer, D. A. (2005). Spectroscopic Properties of Cool Stars (SPOCS). I. 1040 F, G, and K Dwarfs from Keck, Lick, and AAT Planet Search Programs. Astrophys. J. Suppl. 159, 141-166. doi: 10.1086/430500

van Altena, W. F., Lee, J. T., and Hoffleit, D. (1995). VizieR Online Data Catalog: Yale Trigonometric Parallaxes Preliminary (van Altena+ 1991). VizieR Online Data Catal. 1174

van Leeuwen, F. (2007). Validation of the new Hipparcos reduction. Astron. Astrophys. 474, 653-664. doi: 10.1051/0004-6361:20078357

Verbunt, F., and van Gent, R. H. (2012). The star catalogues of Ptolemaios and Ulugh Beg. Machine-readable versions and comparison with the modern Hipparcos Catalogue. Astron. Astrophys. 544:A31. doi: 10.1051/0004-6361/201219596

Ward, J. L., and Kruijssen, J. M. D. (2018). Not all stars form in clusters - measuring the kinematics of OB associations with Gaia. Month. Notic. R. Astron. Soc. 475, 5659-5676. doi: 10.1093/mnras/sty117

Wilkinson, M. I., Vallenari, A., Turon, C., Munari, U., Katz, D., Bono, G., et al. (2005). Spectroscopic survey of the Galaxy with Gaia- II. The expected science yield from the Radial Velocity Spectrometer. Month. Notices R. Astron. Soc. 359, 1306-1335. doi: 10.1111/j.1365-2966.2005.09012.x

Yamada, Y., Fujita, S., Gouda, N., Kobayashi, Y., Hara, T., Nishi, R., et al. (2013). "Scientific goals of Nano-JASMINE", in Advancing the Physics of Cosmic Distances, Vol. 289 of IAU Symposium ed R. de Grijs 429-432.

Conflict of Interest Statement: The author declares that the research was conducted in the absence of any commercial or financial relationships that could be construed as a potential conflict of interest.

The handling Editor declared a shared affiliation, though no other collaboration, with the author AV.

Copyright (c) 2018 Vallenari. This is an open-access article distributed under the terms of the Creative Commons Attribution License (CC BY). The use, distribution or reproduction in other forums is permitted, provided the original author(s) and the copyright owner are credited and that the original publication in this journal is cited, in accordance with accepted academic practice. No use, distribution or reproduction is permitted which does not comply with these terms. 